\title{
SARS-CoV-2 in infant urine and fecal samples after in utero COVID-19 exposure
}

\author{
Jeffery O. Boateng ${ }^{1}$, Elisha M. Wachman ${ }^{1}$, Jacquelyn Turcinovic ${ }^{2}$, Jean Devera ${ }^{3}$, Mayuri Jain ${ }^{4}$, Sigride Jean-Sicard ${ }^{1}$, Elizabeth Woodard ${ }^{1}$, \\ Alice Cruikshank ${ }^{1}$, Bharati Sinha ${ }^{1}$, Ruby Bartolome ${ }^{1}$, Elizabeth D. Barnett ${ }^{1}$, Margaret G. Parker ${ }^{1}$, Christina Yarrington $^{5}$, John H. Connor ${ }^{2}$, \\ Elizabeth Taglauer $^{1}$ and Vishakha Sabharwal ${ }^{1 凶}$
}

(c) The Author(s), under exclusive licence to the International Pediatric Research Foundation, Inc 2021

BACKGROUND: Coronavirus disease 2019 (COVID-19) is a pandemic that has and will continue to affect many pregnant women. Knowledge regarding the risk of vertical transmission is limited. Severe acute respiratory syndrome coronavirus 2 (SARS-CoV-2) realtime reverse transcriptase-polymerase chain reaction (RT-PCR) of nasopharyngeal swabs typically have been used to confirm the diagnosis among infants, but whether the virus can be detected in other biological specimens, and therefore potentially transmitted in other ways, is unknown. Positive SARS-CoV-2 RT-PCR has been reported from feces and urine from adult patients. We hypothesize that the presence of SARS-CoV-2 in infant urine and fecal samples after prenatal COVID-19 exposure is low.

METHODS: We examined the presence of SARS-CoV-2 RNA using RT-PCR in urine and fecal samples among 42 infants born to SARS-CoV-2-infected mothers during different stages of pregnancy.

RESULTS: A urine sample was collected from 39 of 42 infants and fecal samples from all 42 infants shortly after birth. Although the majority of the women had the symptomatic disease (85.6\%), we were unable to detect the presence of SARS-CoV-2 virus from any infant urine or fecal samples.

CONCLUSIONS: SARS-CoV-2 was not detected in infant urine or feces after maternal infection during pregnancy, providing further evidence for low rates of perinatal transmission.

Pediatric Research (2022) 92:536-540; https://doi.org/10.1038/s41390-021-01822-x

\section{IMPACT:}

- SARS-CoV-2 was not detected in the urine or feces of infants of mothers with COVID-19 during various time points in pregnancy.

- This study provides further evidence for low rates of perinatal transmission of SARS-CoV-2.

- Results help to provide guidance on perinatal care practices for infants exposed to COVID-19 in utero.

\section{INTRODUCTION}

Globally, the coronavirus disease 2019 (COVID 19) pandemic has affected millions of pregnant women to date with increased risk for severe illness and concern for mother-to-child transmission of severe acute respiratory syndrome coronavirus 2 (SARS-CoV-2). ${ }^{1}$ The evidence for SARS-CoV-2 being transmitted perinatally is limited, with transmission happening either in utero, intrapartum, or in the early postnatal period. ${ }^{2}$ There are few case reports of in utero transmission of influenza, but no convincing evidence for other respiratory viruses such as SARS-CoV or Middle East respiratory syndrome-related coronavirus. ${ }^{3}$

Even though the presence of SARS-CoV-2 among infants born to mothers with active COVID-19 infection during pregnancy has been studied extensively in the past year, most studies show that positive testing for SARS-CoV-2 among infants is rare. However, testing has been largely focused on reverse transcriptasepolymerase chain reaction (RT-PCR) testing of nasopharyngeal (NP) samples in infants but other relevant biological specimens like infant's urine and feces have not been explored widely. In fecal samples of infected adults, SARS-CoV-2 RNA shedding was seen in $43 \%(934 / 2149)$ of samples. ${ }^{4}$ SARS-CoV-2 has also been detected in vaginal secretions (or fluids) in pregnant women at the time of delivery (five cases reported). ${ }^{5}$ However, in a case report of a possible vertical transmission of SARS-CoV-2 from an infected mother to the infant, maternal vaginal PCR tested negative for SARS CoV-2. ${ }^{6}$ Fecal contamination of the vaginal canal/vulva during labor and childbirth could potentially allow SARS-CoV-2 viral contamination of the neonatal oro/nasopharynx during vaginal birth. ${ }^{7}$ During labor and childbirth or immediately after birth, there may be viral contamination in the environment, due to droplets and aerosols generated by infected women during active labor, as well as maternal fecal contamination, particularly during vaginal birth, which can lead to viral infection in the neonate immediately following birth. ${ }^{8}$ This introduces the challenge of being able to distinguish infant viral infection during passage through the birth canal from a horizontal viral infection of SARS-CoV-2 in the immediate postnatal period when just nasal swab PCR testing in infants is performed. Unlike fecal specimens,

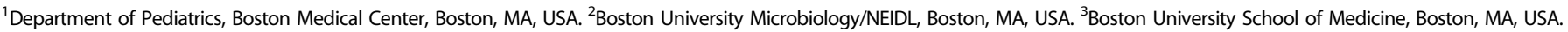

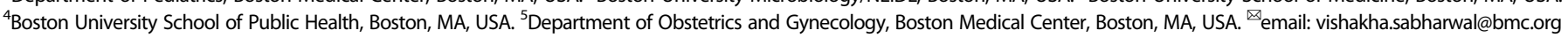


the possibility of transmission via urine is relatively lower given the small amounts of the viral component present (ca. $10^{2}-10^{5}$ genome copies $/ \mathrm{mL}$ ) in urine. ${ }^{9}$ In a study of 72 adults with symptomatic COVID-19 disease, none of the urine samples tested positive for SARS-CoV-2 virus by RT-PCR. ${ }^{10}$

Given the low incidence of neonatal SARS-CoV-2 test result positivity after in utero exposure, identifying the risk factors associated with perinatal transmission has been challenging. ${ }^{11,12}$ In this pilot study, we examined urine and fecal samples of 42 infants born to mothers with positive SARS-CoV-2 tests during pregnancy to evaluate for the presence of SARS-CoV-2 in urine and stool of infants after exposure to SARS-CoV-2 in utero.

\section{METHODS}

\section{Study design and patient population}

Boston Medical Center (BMC) is the largest urban safety-net hospital in New England serving most of the underrepresented minority populations living in the Greater Boston Area. The hospital has 3000 births per year in the Labor and Delivery Unit with the majority of women being from minority groups with comorbidities such as hypertension and obesity and having public insurance. These characteristics have been associated with severe SARS-COV-2 disease. ${ }^{13,}{ }^{14}$ BMC performed universal screening of all women who presented in labor since April 2020. During the enrollment period, BMC attended to 157 mothers who tested positive for SARS-CoV-2 at any point in pregnancy.

Between the period of July 2020 and February 2021, we enrolled mother-infant dyads from the obstetric prenatal clinics, Labor and Delivery Unit, and the Postpartum Unit in a prospective cohort study. To be eligible, mothers had to be at least 18 years of age, have a documented positive SARS-CoV-2 infection at any point during pregnancy as documented by nasal swab PCR testing, have a viable singleton gestation pregnancy, be English or Spanish speaking, and plan on delivering at BMC. Mothers were excluded if they were admitted to the intensive care unit (ICU) at the time of approach for consent, unable to provide consent, or are unlikely to keep custody of their infant. Written informed consent was obtained from all subjects. This study was approved by the Boston University Medical Campus Institutional Review Board.

\section{Sample collection}

Within the first 3 days after delivery, fecal and urine samples for the study were collected from the infants by bedside nurses and trained study staff in coordination with routine care. Fecal samples were obtained by dipping a calcium alginate swab in any stool in the diaper or when there was no stool in the diaper, swabbing the external anal area gently but thoroughly. Swabs were then cut and placed into a $2 \mathrm{~mL}$ cryovial containing viral media solution. The swab with fecal specimen was then stored at $-80^{\circ} \mathrm{C}$ within $6 \mathrm{~h}$ of collection. Urine samples were collected by placing an absorbent diaper or cotton balls in the infant's diaper with a goal of obtaining $1-5 \mathrm{~mL}$ of urine per infant. Urine was then stored at $-80^{\circ} \mathrm{C}$ within $6 \mathrm{~h}$ of collection.

\section{Laboratory methods}

Frozen urine samples and fecal swabs were thawed, and total RNA from $300 \mu \mathrm{L}$ of each sample was extracted according to the Zymo Quick-RNA Viral Kit protocol (Zymo Research Corp., Irvine, CA, 2021). RNA was eluted into $15 \mu \mathrm{L}$ of nuclease-free water, after which quantitative reverse transcription-PCR (RT-qPCR) was performed using $1 \mu \mathrm{L}$ of RNA and the iTaq Universal SYBR ${ }^{\oplus}$ Green One-Step Kit (Rio-Rad Laboratories Inc., Hercules, CA, 2021). The CDC N1 primer (Integrated DNA Technologies, Coralville, IA, 2021) was used for amplification and quantification of SARS CoV-2 genomic RNA, and each reaction ran for 40 amplification cycles. In vitro transcribed RNA standards ranging from $10^{9}$ to $10^{3}$ genome copies $/ \mu \mathrm{L}$ were used for quantification, and two non-template controls were included in each run. Our laboratory ran control infant urine and rectal swab samples spiked with in vitro transcribed SARS-CoV-2 genomic RNA, which validated our RT-qPCR assay prior to running our study samples (Supplemental Fig. 1).

\section{Data abstraction}

The electronic medical records of the mother-infant dyads enrolled were reviewed and abstracted. Demographic characteristics such as race, ethnicity, age at delivery, and primary language were recorded in a secure database. Other maternal data points such as the history of chronic illnesses, pregnancy-related diagnosis, and SARS-CoV-2 infection-related symptoms were collected. Trimester and gestational age at the time of infection, the severity of infection (CDC criteria/ICU admission), and maternal COVID-19 status at the time of delivery were also collected. For infants, demographics and birth parameters as well as COVID-19 testing status were reviewed. Infants were also tracked for any emergency room visits or readmissions in the first 30 days of life using electronic chart review.

\section{Statistical methods}

Demographic characteristics, pregnancy maternal outcomes, and neonatal outcomes were summarized for the mother-infant dyads. Categorical variables were reported as percentages $[n(\%)]$ and continuous variables were reported as either mean with standard deviation [mean (SD)] or median with interquartile range [median (IQR)], where appropriate. The descriptive analyses summarized in Tables 1 and 2 were conducted using SAS 9.4 (SAS Institute Inc., Cary, NC, 2013).

\section{RESULTS}

A total of 103 mothers with positive SARS-CoV-2 PCR NP swabs were screened for eligibility during the recruitment period of 1 July 2020 to 8 February 2021. Of these, 87 mother-infant dyads were deemed eligible, out of which 80 were approached for consent. Forty-two (52\%) consented to participate in the study and all mothers who consented were included in the study with no exclusions after delivery (Fig. 1).

Demographics of the 42 mother-infant dyads are shown in Tables 1 and 2. The majority (78.6\%) of participants were of Hispanic ethnicity. Many of the mothers had at least one chronic health condition, such as diabetes $(7.1 \%)$ or obesity $(9.5 \%)$, or a pregnancy comorbidity such as hypertensive disorder of pregnancy (26.2\%) (Table 1). Thirty-eight percent delivered via cesarean section. Trimester of COVID-19 infection varied, with 7 first trimester (16.7\%), 17 second trimester $(40.5 \%)$, and 18 third trimester (42.9\%) infections. Only six of 18 women in the third trimester had a positive SARS-CoV-2 PCR at the time of delivery with only 2 being symptomatic; all others had recovered by the time of delivery. The six infants born to mothers with active disease were evaluated and had a negative nasopharyngeal SARSCoV-2 PCR at 24 and $48 \mathrm{~h}$ after birth per American Academy of Pediatrics (AAP) guidelines. ${ }^{15}$ Eighty-five percent of mothers reported COVID-19 symptoms at the time of PCR-confirmed infection, and four (9.5\%) required hospitalization for treatment of COVID-19 complications. All of the infants were born $\geq 35$ weeks' gestational age (range 35.3-41.5 weeks) (Table 2).

A urine sample was collected from 39 of 42 infants and a fecal sample from all 42 infants in the study. Urine samples were missed from three infants due to study staff availability related to vaginal deliveries with a more limited time window for sample collection. For the remaining 39 infants, urine samples were collected at a mean of $32 \mathrm{~h}$ (SD 6) after delivery. Fecal samples were collected at a mean of $15 \mathrm{~h}$ (SD 6) after delivery. Results of RT-PCR testing for both urine and fecal samples were all negative. Two infants had emergency room visits. One was on account of transient fast breathing, and the other was due to hypothermia in a preterm infant requiring admission for suspected neonatal sepsis. Both infants tested negative for COVID-19 nasal PCR at the time of their emergency room visits.

\section{DISCUSSION}

At a single center with a high incidence of SARS-CoV-2 infection during pregnancy, we examined infant fecal and urine samples for the presence of SARS-CoV-2. We did not find evidence of SARSCoV-2 infection among infants in any bodily sample, even among those born to mothers with active SARS-CoV-2 infection at the 
Table 1. Demographics and outcomes of 42 COVID-19-positive pregnant women.

\begin{tabular}{|c|c|}
\hline Characteristic & $N(\%)$ or mea \\
\hline \multicolumn{2}{|l|}{ Race $^{a}$} \\
\hline Black & $6(14.3 \%)$ \\
\hline White & $9(21.4 \%)$ \\
\hline Asian & $1(2.4 \%)$ \\
\hline Unknown & $4(9.5 \%)$ \\
\hline Other & $22(52.4 \%)$ \\
\hline \multicolumn{2}{|l|}{ Ethnicity $^{\mathrm{a}}$} \\
\hline Hispanic & $33(78.6 \%)$ \\
\hline Non-Hispanic & $9(21.4 \%)$ \\
\hline \multicolumn{2}{|l|}{ Language $^{a}$} \\
\hline English & $20(47.6 \%)$ \\
\hline Spanish & $22(52.4 \%)$ \\
\hline Age at delivery (years) & $31.3(6.2)$ \\
\hline \multicolumn{2}{|l|}{ Chronic health conditions ${ }^{\mathrm{b}}$} \\
\hline Diabetes & $3(7.1 \%)$ \\
\hline Hepatitis C virus & $1(2.4 \%)$ \\
\hline Herpes simplex virus & $2(4.8 \%)$ \\
\hline Hypertension & $2(4.8 \%)$ \\
\hline Obesity & $4(9.5 \%)$ \\
\hline Thyroid disease & $1(2.4 \%)$ \\
\hline Substance use disorder & $3(7.1 \%)$ \\
\hline \multicolumn{2}{|l|}{ Pregnancy comorbidities ${ }^{b}$} \\
\hline Chorioamnionitis & $7(16.7 \%)$ \\
\hline Gestational diabetes & $7(16.7 \%)$ \\
\hline Hypertensive disorder of pregnancy & $11(26.2 \%)$ \\
\hline \multicolumn{2}{|l|}{ Delivery mode } \\
\hline Vaginal delivery & $26(61.9 \%)$ \\
\hline Cesarean Section & $16(38.1 \%)$ \\
\hline \multicolumn{2}{|l|}{ Trimester of COVID-19 infection ${ }^{c}$} \\
\hline First & $7(16.7 \%)$ \\
\hline Second & $17(40.5 \%)$ \\
\hline Third & $18(42.9 \%)$ \\
\hline Symptomatic infection ${ }^{d}$ & $36(85.7 \%)$ \\
\hline $\begin{array}{l}\text { 1. Confirmed SARS-CoV-2 with active } \\
\text { symptoms at delivery }\end{array}$ & $2(4.8 \%)$ \\
\hline $\begin{array}{l}\text { 2. Confirmed SARS-CoV-2 and asymptomatic } \\
\text { at delivery }\end{array}$ & $4(9.5 \%)$ \\
\hline $\begin{array}{l}\text { 3. Recovered fromSARS-CoV-2 infection } \\
\text { earlier in pregnancy }\end{array}$ & $36(85.7 \%)$ \\
\hline Hospitalized for COVID-19e & $4(9.5 \%)$ \\
\hline
\end{tabular}

${ }^{a}$ Race/ethnicity and language were self-identified by participants and confirmed with medical records and when a discrepancy was identified, the self-identified demographics were used.

${ }^{b}$ Chronic illnesses and pregnancy comorbidities were defined as any physician-diagnosed condition as recorded in the medical records of participants and for which they are receiving management.

'Trimester of infection is defined as the trimester when the mother had a positive NP PCR test.

${ }^{\mathrm{d}}$ Symptomatic infection is defined as mothers who reported at least one COVID-19 symptom per CDC criteria with a positive NP PCR test.

eHospitalized for COVID-19 is defined as the history of admission to the hospital for management of COVID-19-related symptoms at any point in pregnancy other than delivery admission. time of birth. This corroborates previous studies demonstrating a low rate of SARS-CoV-2 positive testing among infants, but extends upon past literature by additionally examining urine and fecal samples.

Overall, the majority of COVID-19 cases of pregnant women with fetal or neonatal outcomes reported thus far involved patients in their third trimester of pregnancy. In a small case series of 19 women who were symptomatic at the time of delivery carried out in China where the neonates were immediately separated from the mothers after delivery and isolated, ${ }^{16}$ SARSCoV-2 RT-PCR in throat swab, urine, and feces of all neonates were performed and were negative. They even tested for SARS-CoV-2 RT-PCR in breast milk and amniotic fluid, which was negative. None of these 19 neonates developed any clinical or radiologic evidence of COVID-19 and acted appropriately for gestational age. The results of this small study did not support the intrauterine vertical transmission of SARS-CoV-2.

In another study from China, which included 33 neonates born to mothers with COVID-19, three neonates were identified to have COVID-19, the nasopharyngeal and anal swabs were positive for SARS-CoV-2 on days 2 and 4 of life and negative on day 6 and the most common symptom was shortness of breath and all infants did well. ${ }^{17}$ In our study, we were unable to detect any SARS-CoV-2 virus in either the urine or fecal samples of exposed infants, implying these infants were not infected in utero or perinatally. These results in conjunction with the two previously described studies provide evidence that the likelihood of perinatal transmission in these infants was low. All newborns exposed to maternal SARS-CoV-2 were in good condition at birth and all of them were asymptomatic and appropriate for gestational age.

The emerging evidence suggests low rates of virus positivity in the nasopharynx, but other relevant biological specimens like infant's urine and feces have not been explored widely. ${ }^{10,} 18$ Low rates of virus positivity in other biological specimens like the placenta and breast milk suggest that perinatal transmission is uncommon. $^{19,20}$ At the height of the pandemic in 2020, clinical practices that were recommended to reduce perinatal transmission included elective cesarean delivery, mother-newborn separation, and temporary suspension of breastfeeding. ${ }^{21}$ Subsequently, based on accumulating evidence, the AAP promoted rooming-in and breastfeeding with precautions. ${ }^{15} \mathrm{~A}$ couple of more recent

Table 2. Demographics and outcomes of 42 COVID-19 exposed neonates.

\begin{tabular}{|c|c|}
\hline Characteristic & $N(\%)$ or median (IQR) \\
\hline Gestational age (weeks) & $39.3[37.6-40.5]$ \\
\hline Birth weight (g) & $3320[2980-3605]$ \\
\hline \multicolumn{2}{|l|}{ Sex } \\
\hline Female & $23(54.8 \%)$ \\
\hline Male & $19(45.2 \%)$ \\
\hline Length of hospital stay (days) ${ }^{a}$ & $3[2-4]$ \\
\hline Received breastmilk & $39(92.9 \%)$ \\
\hline Emergency room visit within 30 days $^{\mathrm{b}}$ & $2(4.8 \%)$ \\
\hline Hospital re-admission within 30 days $^{\mathrm{b}}$ & $1(2.4 \%)$ \\
\hline \multicolumn{2}{|c|}{$\begin{array}{l}\text { aLength of stay is defined as the number of days a neonate spent in the } \\
\text { hospital after delivery. } \\
\text { b Of the two ED visits, one was on account of a transient episode of rapid } \\
\text { breathing with no further symptoms who was discharged the same day. } \\
\text { The second ED visit was preterm at } 35 / 4 \text { weeks presented on day } 4 \text { of life } \\
\text { with hypothermia. This visit ended in an admission when the neonate was } \\
\text { managed for sepsis rule out and discharged after } 3 \text { days. They both tested } \\
\text { negative for COVID- } 19 \text { by NP-PCR at presentation. }\end{array}$} \\
\hline
\end{tabular}




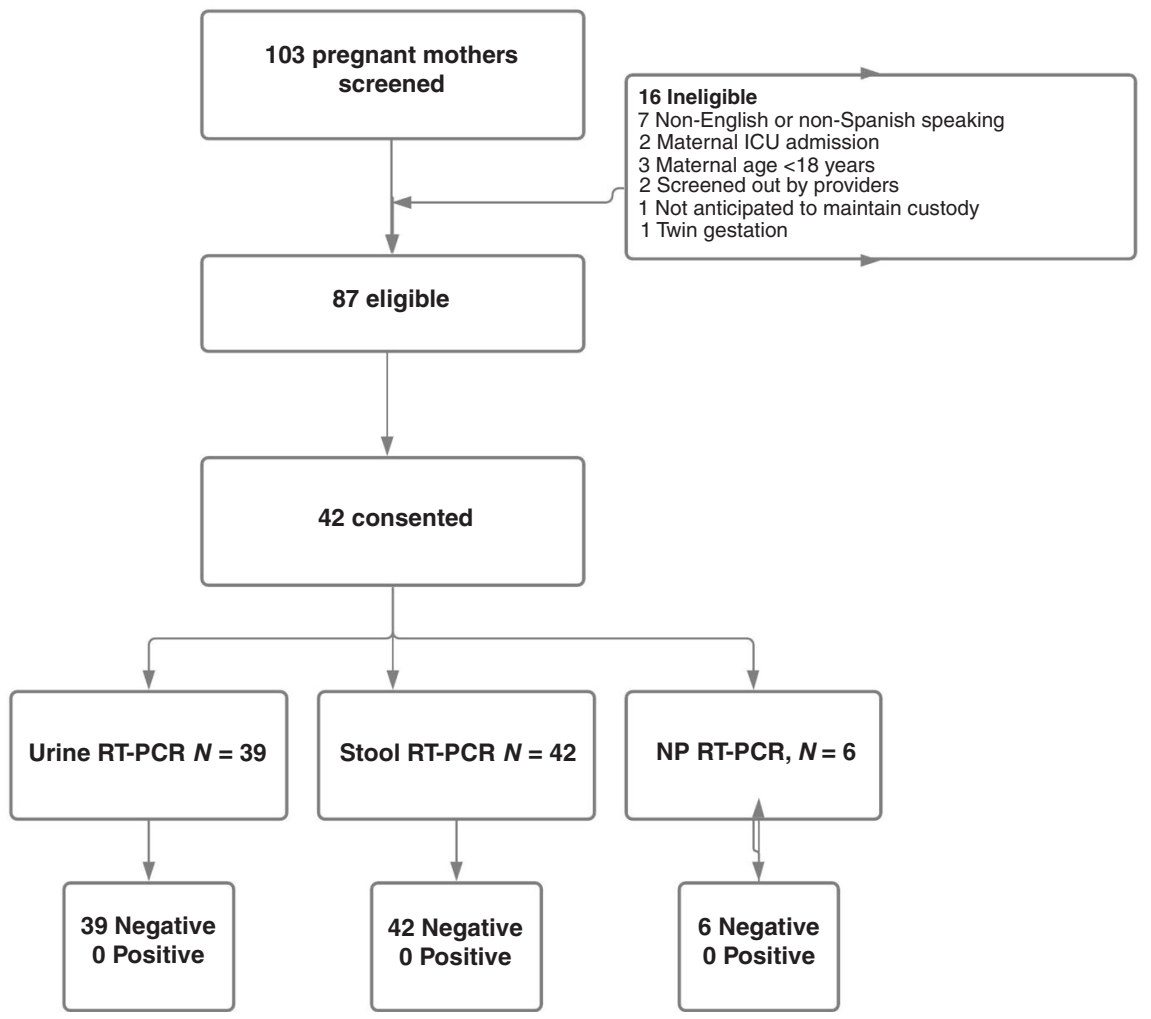

Fig. 1 Screening, enrollment, and results of Urine, Stool, and Nasopharyngeal SARS CoV-2 PCR.

large prospective cohort studies of SARS-CoV-2 infection in pregnancy, the authors did not find evidence of placental infection or definitive vertical transmission of SARS-CoV-2. ${ }^{22,} 23$ Another study from New York found two infants with indeterminate tests, but concluded that there was no clinical evidence of vertical transmission in 101 newborns of mothers positive for or with suspected SARS-CoV-2 infection, despite most newborns rooming-in and direct breastfeeding practices. ${ }^{3}$ The experience at our institution as opposed to other studies, where amongst 75 pregnant women with SARS-CoV-2-positive testing, five infants (7\%) had one or more SARS-CoV-2 positive tests in the first week of life, which we attribute to severe maternal illness at the time of delivery. ${ }^{24}$ Of note, there was no overlap with the infants included in our prior study with the present study.

The real strength and novel aspect of this study include the examination of neonatal SARS-CoV-2 nucleic acid testing of neonatal fecal and urine samples. We also included pregnant women in different stages of pregnancy and followed the infants until 30 days of age.

Limitations of our study include our small sample size, although it was a pilot study. Also, given that we collected specimens from infants born to mothers infected at different stages of pregnancy, we did not have nasopharyngeal specimens for SARS-CoV-2 PCR on all 42 infants in the study for comparison. Since our study only enrolled mothers with documented SARS-CoV-2 infection, we also did not have PCR results from control mother-infant dyads without SARS-CoV-2 infection for comparison. However, our laboratory did run control samples spiked with in vitro transcribed SARS-CoV-2 genomic RNA, which confirmed that our RT-qPCR assay would have amplified viral RNA if it was present in the infants' urine or rectal samples. Also, we did not look at SARS-CoV-2 nucleic acid detection in other biological specimens such as breast milk, cord blood, placenta, or amniotic fluid. These additional biological specimens could be evaluated in future studies.

In conclusion, we assessed for the presence of SARS-CoV-2 in urine and feces of infants of mothers infected at different times during pregnancy and did not detect SARS-CoV-2 in any of the samples. This confirms other studies and supports the evidence for low rates of perinatal transmission of SARS-CoV-2. Future studies looking at the detection of SARS-CoV-2 nucleic acid detection of breast milk, cord blood, placenta, and amniotic fluid are needed to further evaluate the presence of SARS CoV-2 in infants born to mother infected during the first, second, and third trimester as well at the time of delivery.

\section{REFERENCES}

1. Allotey, J. et al. Clinical manifestations, risk factors, and maternal and perinatal outcomes of coronavirus disease 2019 in pregnancy: living systematic review and meta-analysis. BMJ 370, m3320 (2020).

2. Dumitriu, D. et al. Outcomes of neonates born to mothers with severe acute respiratory syndrome coronavirus 2 infection at a large medical center in New York City. JAMA Pediatr. 175, 157-167 (2021).

3. Schwartz, D. A. \& Dhaliwal, A. Infections in pregnancy With COVID-19 and other respiratory RNA Virus diseases are rarely, if ever, transmitted to the fetus: experiences with coronaviruses, parainfluenza, metapneumovirus respiratory syncytial virus, and influenza. Arch. Pathol. Lab. Med. 144, 920-928 (2020).

4. van Doorn, A. S., Meijer, B., Frampton, C. M. A., Barclay, M. L. \& de Boer, N. K. H. Systematic review with meta-analysis: SARS-CoV-2 stool testing and the potential for faecal-oral transmission. Aliment. Pharmacol. Ther. 52, 1276-1288 (2020).

5. Yap, M. et al. Clinical manifestations, prevalence, risk factors, outcomes, transmission, diagnosis and treatment of COVID-19 in pregnancy and postpartum: a living systematic review protocol. BMJ Open 10, e041868 (2020).

6. Dong, L. et al. Possible vertical transmission of SARS-CoV-2 from an infected mother to her newborn. JAMA 323, 1846-1848 (2020).

7. Carosso, A. et al. Pre-labor anorectal swab for SARS-CoV-2 in COVID-19 pregnant patients: is it time to think about it? Eur. J. Obstet. Gynecol. Reprod. Biol. 249, 98-99 (2020).

8. Hermesch, A. C. et al. Severe acute respiratory syndrome coronavirus 2 (SARSCoV-2) environmental contamination and childbirth. Obstet. Gynecol. 136, 827-829 (2020).

9. Jones, D. L. et al. Shedding of SARS-CoV-2 in feces and urine and its potential role in person-to-person transmission and the environment-based spread of COVID19. Sci. Total Environ. 749, 141364 (2020). 
10. Wang, W. et al. Detection of SARS-CoV-2 in different types of clinical specimens. JAMA 323, 1843-1844 (2020).

11. Sheth, S., Shah, N. \& Bhandari, V. Outcomes in COVID-19 positive neonates and possibility of viral vertical transmission: a narrative review. Am. J. Perinatol. 37, 1208-1216 (2020).

12. Fajnzylber, J. et al. SARS-CoV-2 viral load is associated with increased disease severity and mortality. Nat. Commun. 11, 5493 (2020).

13. Gupta, S. et al. Factors associated with death in critically ill patients with coronavirus disease 2019 in the US. JAMA Intern. Med. 180, 1436-1447 (2020).

14. Aldridge, R. W. et al. Black, Asian and Minority Ethnic groups in England are at increased risk of death from COVID-19: indirect standardisation of NHS mortality data. Wellcome Open Res. 5, 88 (2020).

15. FAQs: management of infants born to mothers with suspected or confirmed COVID-19. http://www.aap.org/en/pages/2019-novel-coronavirus-covid-19-infections/ clinical-guidance/faqs-management-of-infants-born-to-covid-19-mothers/ (2021)

16. Liu, W. et al. Clinical characteristics of 19 neonates born to mothers with COVID19. Front. Med. 14, 193-198 (2020)

17. Zeng, L. et al. Neonatal early-onset infection with SARS-CoV-2 in 33 neonates born to mothers with COVID-19 in Wuhan, China. JAMA Pediatr. 174, 722-725 (2020).

18. Fenizia, C. et al. Analysis of SARS-CoV-2 vertical transmission during pregnancy. Nat. Commun. 11, 5128 (2020).

19. Chambers, C. et al. Evaluation for SARS-CoV-2 in breast milk from 18 infected women. JAMA 324, 1347-1348 (2020).

20. Vivanti, A. J. et al. Transplacental transmission of SARS-CoV-2 infection. Nat. Commun. 11, 3572 (2020).

21. Chen, D. et al. Expert consensus for managing pregnant women and neonates born to mothers with suspected or confirmed novel coronavirus (COVID-19) infection. Int. J. Gynecol. Obstet. 149, 130-136 (2020).

22. Edlow, A. G. et al. Assessment of maternal and neonatal SARS-CoV-2 viral load, transplacental antibody transfer, and placental pathology in pregnancies during the COVID-19 pandemic. JAMA Netw. Open 3, e2030455 (2020).

23. Salvatore, C. M. et al. Neonatal management and outcomes during the COVID-19 pandemic: an observation cohort study. Lancet Child Adolesc. Health 4, 721-727 (2020).

24. Sabharwal, V. et al. Mother-infant dyads with COVID-19 at an urban, safety net hospital: clinical manifestations and birth outcomes. Am. J. Perinatol. 38, 741-746 (2021).

\section{ACKNOWLEDGEMENTS}

We would like to acknowledge all of the bedside nurses, midwives, and physicians from the Boston Medical Center Labor and Delivery Unit, Postpartum Unit, and Newborn
Intensive Care Unit who assisted us with subject recruitment and facilitation of sample collection, especially Kate Thibault, RN; Lauren Laliberte, RN; Brianna Medeiros, RN, NP; Elizabeth Regan, RN, and Joanna Bushfield, RN. We would also like to acknowledge the Maxwell Finland Laboratory of Pediatric Infectious Disease at Boston Medical Center and the Boston University NEIDL laboratory of microbiology.

\section{AUTHOR CONTRIBUTIONS}

J.O.B., E.M.W., and V.S. created the first draft of the manuscript. J.O.B., E.M.W., V.S., J.T., J.D., M.J., E.D.B., M.G.P., C.Y., J.H.C., and E.T. made substantial contributions to conception and design, acquisition of data, analysis, and interpretation of data. S.J.-S., E.W., A.C., B.S., and R.B. contributed to the acquisition of data. All authors critically revised and revised the article for important intellectual consent and approved the final version to be published.

\section{FUNDING}

This project was supported by funding from the Boston University Clinical and Translational Science Institute (1UL1TR001430).

\section{COMPETING INTERESTS}

The authors declare no competing interests.

\section{CONSENT STATEMENT}

Written informed consent was obtained from all participants in the study.

\section{ADDITIONAL INFORMATION}

Supplementary information The online version contains supplementary material available at https://doi.org/10.1038/s41390-021-01822-x.

Correspondence and requests for materials should be addressed to Vishakha Sabharwal.

Reprints and permission information is available at http://www.nature.com/ reprints

Publisher's note Springer Nature remains neutral with regard to jurisdictional claims in published maps and institutional affiliations. 